\title{
Electromyographic patterns in adult locomotion: A comprehensive review
}

\author{
RICHARD SHIAVI, Ph.D. \\ Department of Electrical and Biomedical Engineering, Vanderbilt University; \\ and Research Service, Veterans Administration Medical Center, Nashville, Tennessee
}

\section{INTRODUCTION}

Investigations of muscular synergy patterns in locomotion have had a variety of purposes, and the results have been presented in different degrees of detail. Some studies focused on muscles acting on a particular joint, whereas others considered selected muscle groups of the lower extremity. Partial summaries appear in the major literature on the kinesiology of gait. Included are the works of Eberhart et al. (10), Basmajian ( $\%$ ), Perry (23), and Inman et al. (16). The book by Inman et al. (16) contains an excellent pictorial representation of general muscle function, differentiating eccentric and concentric action. An example of the classic results is shown in Figure 1.

Population variabilities in electromyographic (EMG) patterns have been noted, and more recently, attention has been directed toward this fact $(1,19,31,36)$. Paul (21) has compiled the results of several reported investigations and indicated some significant inconsistencies on the phasings of particular muscles or groups. These apparent disagreements may be attributed either to differences in investigatory protocol or to actual inter-individual dynamic variations (22). For instance, it is known that EMG patterns can change with walking speed $(19,34)$. The preference of the investigator or the size and depth of the muscles or groups also influenced whether surface or intramuscular electrodes were used to record EMG activity. The investigatory para- digms, as well as the results presented in terms of the action of either groups or specific muscles, differed generally. Some investigators stated that muscles in a group had patterns similar enough to average their patterns, whereas others stated the opposite. For instance, compare the statements regarding EMG patterns of thigh musculature made by Battye and Joseph (4) with those made by Grieve and Cavanagh (14). At the other extreme, noted investigators such as Basmajian (3) and Inman (16) state that different regions in some muscles produce different EMG patterns. The principal differences in experimental protocol are whether 1) subjects walked freely or in cadence with a metronome; 2) subjects walked at their free speed or other speed; 3) subjects wore shoes or were barefooted; 4) subjects walked on a floor, metal plate, or treadmill; or, 5) EMG activity was recorded with surface, wire, or needle electrodes.

Another factor that complicates the assimilation of the results of all the investigations is the different methods used to analyze and represent EMG activity. This also hinders the creation of a common database for all investigators. A recent standards report contains a plea for standardization of research results (42). This report and recent publications cogently argue that the best method is the ensemble average of the timenormalized linear envelope $(5,32,43)$. Another significant question is the type of amplitude normalization to reduce inter-subject variability. Again, recent publications show that normaliza- 

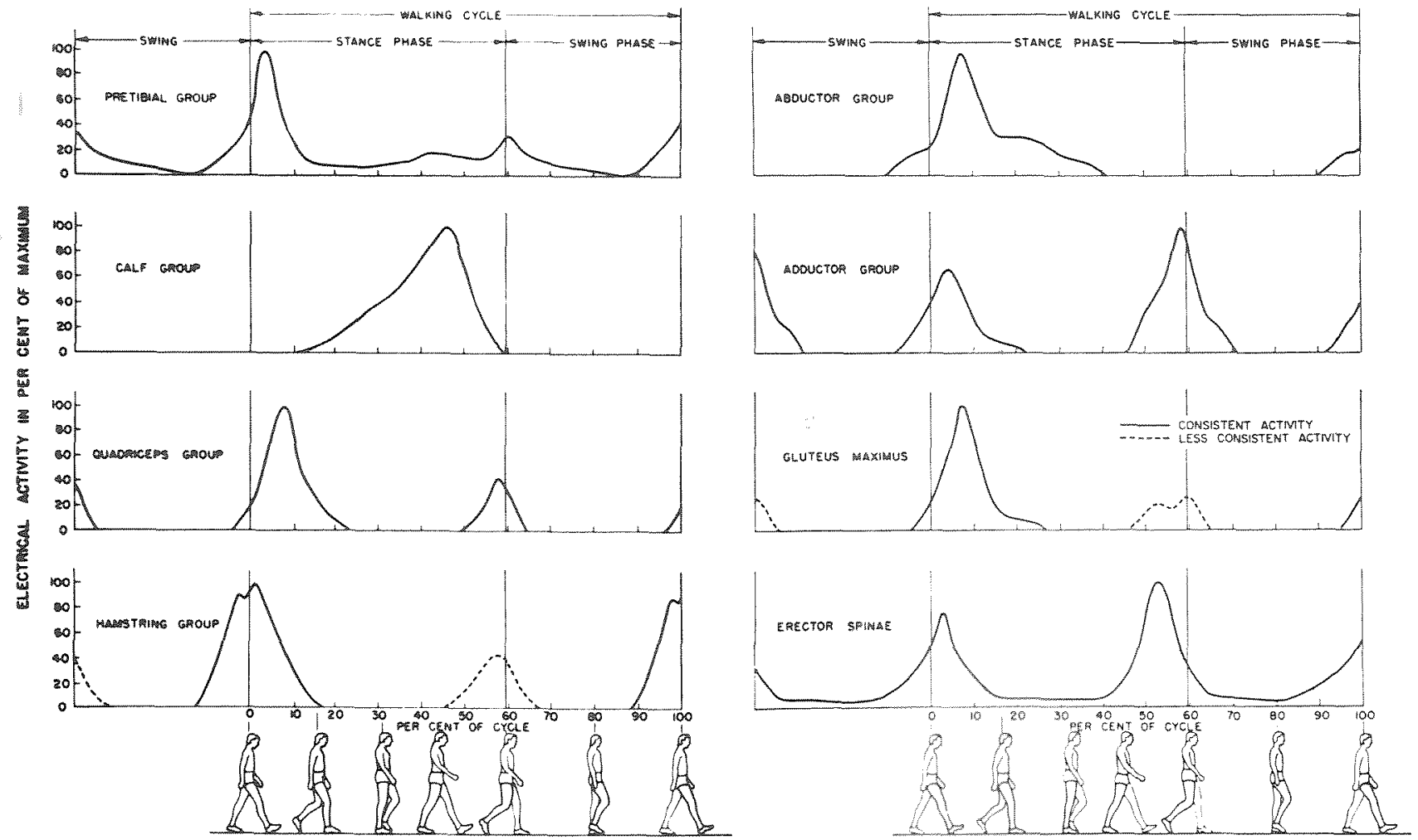

FIGURE 1

Electromyographic (EMG) envelopes of major musele groups. [From Eberhart ot al. (10), ]

tion by the stride average reduces variability the most $(35,44)$.

Besides the linear envelope, investigators in the past have used a variety of representations that include: 1) raw data (3), 2) on-off patterns (23), and 3) full-wave rectified and integrated values in 5 percent stride segments (14).

The significance of the results is also influenced by the number of subjects in any particular investigation. In most investigations less than 14 subjects were studied. The various investigatory features are summarized in Table 1; note that often not all experimental conditions are mentioned. Because of these differences, this review attempts to coalesce all of the known results into an informational base on locomotor EMG in adults. It is not intended to be a kinesiology review, although some of the current concepts are mentioned to provide a more relevant understanding. Before the synergy patterns are reviewed, gait terminology and stride parameters are briefly summarized.

\section{TERMINOLOGY}

The standard descriptive terminology is presented by Perry (24). Each stride (gait cycle) is composed of two steps and is divided into two major phases: 1) the stance phase, which is the time period when any part of the ipsilateral foot is in contact with the walking surface; and 2) the swing phase, which is the time period when that foot is not in contact. The times of occurrence of all the events are normalized to percentages of the stride. Figure 2 depicts a typical normal timing sequence.

The stance phase consists of several periods: 1 ) loading, which is the initial period of double-leg support (DLS) extending from foot-strike to the toe-off of the contralateral foot, 2) midstance, which is a period of single-leg support (SLS) extending from the end of DLS to the time when the body is positioned over the supporting leg (at approximately 24 percent of the stride); 3) terminal stance, which is the remainder of SLS; and 4) unloading or preswing, which is the second 
TABLE 1. Electromyographic measurement of gait

\begin{tabular}{|c|c|c|c|c|c|c|c|c|c|c|}
\hline Rens. & $N$ & Age & Electrode & Footwear & Timing & Speed, $(\mathrm{m} / \mathrm{s})^{i}$ & Surface & EMG Mode & Notes ${ }^{g}$ & Foot Contact \\
\hline 1 & 17 & $21-40$ & $\mathrm{~S}$ & $\mathrm{SH}$ & SS & & & on-off & \multirow{4}{*}{$\begin{array}{c}\text { threshold }=15 \% \\
\text { of peak }\end{array}$} & \multirow{4}{*}{ video } \\
\hline 3 & & & $\mathrm{~W}$ & $\mathrm{SH}$ & SS & $\mathrm{F}$ & $\mathrm{F}$ & raw & & \\
\hline 4 & 14 & $14-48$ & $\mathrm{~S}$ & $\mathrm{SH}$ & $\mathrm{M}$ & & $\mathrm{F}$ & on-off & & \\
\hline 6 & 5 & & S & SN & & $1.1,1.8$ & $\mathrm{~T}$ & $\mathrm{SIC}$ & & \\
\hline$y$ & 20 & $8-72$ & S & SH & M-SS & $\overline{\mathrm{ST}}=1.1$ & $\mathrm{~F}$ & ENV & \multirow[t]{2}{*}{ NBM } & FS \\
\hline 10 & 10 & & $\mathrm{~S}$ & & M & $\overline{\mathrm{ST}}=1.2$ & & ENV & & FS \\
\hline 11 & 20 & students & W & $\mathrm{SH}$ & SS & $\mathrm{F}$ & $F$ & raw & \multirow{4}{*}{ NBM } & FS \\
\hline 12 & 14 & $18-31$ & W & $\mathrm{SN}$ & & 1.22 & $\mathrm{~T}$ & raw & & FS \\
\hline 17 & 11 & $25-34$ & $\mathrm{~W}$ & & SS & $1.3-2.1$ & F & INT & & FS \\
\hline 18 & 12 & $15-25$ & $\mathrm{~W}$ & $\mathrm{SH}$ & SS & $\mathrm{F}$ & M & raw & & FS \\
\hline 19 & 6 & aduit & W & SH & pace & $0.67-2.28$ & M & ENV & \multirow[t]{3}{*}{$\mathrm{NBM}$} & \multirow[t]{3}{*}{ FS } \\
\hline 21 & 14 & & $\mathrm{~S}$ & $\mathrm{SH}$ & SS & $1.4-2.1$ & & on-off & & \\
\hline \multirow[t]{2}{*}{26} & 10 & $20-25$ & $\mathrm{~S}$ & & & & & INT & & \\
\hline & & & & & & $\overline{\mathrm{ST}}=1.55$ & & & & \\
\hline 27 & 10 & $20-33$ & $\mathrm{~N}$ & & M-SS & $\overline{\mathrm{SL}}=110 \mathrm{~cm}$ & $\mathrm{R}$ & raw & graded, 0-5 & video \\
\hline 28 & 10 & & $\mathrm{~S}$ & SH & M & $\mathrm{ST}=2.0$ & $\mathrm{~F}$ & raw & & FS \\
\hline 29 & 10 & & $\mathrm{~S}+\mathrm{N}$ & $\mathrm{SH}$ & M & $\mathrm{ST}=2.0$ & $\mathrm{~F}$ & raw & & FS \\
\hline 31 & 25 & $20-40$ & $\mathrm{~S}$ & $\mathrm{BF}$ & SS & $0.25-1.68$ & $\mathrm{M}$ & on-off & & FS \\
\hline 36 & 8 & $12-36$ & W & $\mathrm{SH}$ & SS & & $\mathrm{F}$ & on-off & & FS \\
\hline 39 & 6 & & W & $\mathrm{SH}$ & SS & $\mathrm{F}$ & F & ENV & NBM & cine \\
\hline 40 & 9 & $20-35$ & $\mathrm{~S}$ & $\mathrm{BF}$ & SS & & $\mathrm{T}$ & raw & & FS \\
\hline 41 & 10 & young & $\tilde{W}$ & $\mathrm{SH}$ & & $1.22,1.47$ & $\mathrm{~T}$ & on-off & & FS \\
\hline
\end{tabular}

Blank blocks indicate no data given in publication. $N=$ number of subjects.

" $\mathrm{S}=$ surface, $\mathrm{W}=$ wire, $\mathrm{N}=$ needle.

${ }^{\mathrm{b}} \mathrm{SH}=$ shoes, $\mathrm{BF}=$ barefoot, $\mathrm{SN}=$ sneakers.

" $\mathrm{SS}=$ self-selected, $\mathrm{M}=$ mecronome.

${ }^{a} \mathrm{~F}=$ free, $\mathrm{ST}=$ stride time $(\mathrm{s})$, overbar $=$ average.

$\mathrm{F}=$ floor, $\mathrm{T}=$ treadmill, $\mathrm{M}=$ metal mat, $\mathrm{R}=$ rubber mat.

ENV = envelope, $\mathrm{INT}=$ integrated, $\mathrm{SIC}=$ spike integrator counts.

NBM = normalized by maximum.

${ }^{h} \mathrm{FS}=$ foot switchers.

period of DLS extending from contralateral footstrike to ipsilateral toe-off. The swing phase is divided into three stages corresponding approximately to the initial (i.e., early), middle, and terminal (i.e., late) time periods. Additional terms are used to account for the fact that muscles are active through most of the two time periods within the transitions between stance and swing phases. Stance-swing transition period comprises unloading and initial swing periods; swing-stance transition period comprises terminal swing and loading periods.

\section{PARAMETERS}

The stride parameters, such as speed, stride length, and stride frequency are important. Dean (7), Grieve (13), and Milner and Quanbury (20), among others, have contributed to the knowledge of these essential parameters. The unanimous conclusion is that humans are capable of an eightfold variation in walking speed by varying both stride frequency and length. The speed of progression is made faster by increases in both. At the faster walking speeds, a maximum limit on stride length is reached; the fastest speeds are achievable by increasing only stride frequeney. Nominal values of self-selected speeds in the laboratory for slow, free, and fast walking speeds are $0.68,0.98$, and 1.39 meters per second, respectively (31). As Drillis (8) has remarked: "a laboratory setting can induce shorter step lengths, thus causing slower speeds."

\section{MUSCULAR SYNERGY PATTERNS}

This review discusses EMG patterns modularly, according to anatomic regions. Discussions are 


\section{TYPICAL NORMAL WALK CYCLE}

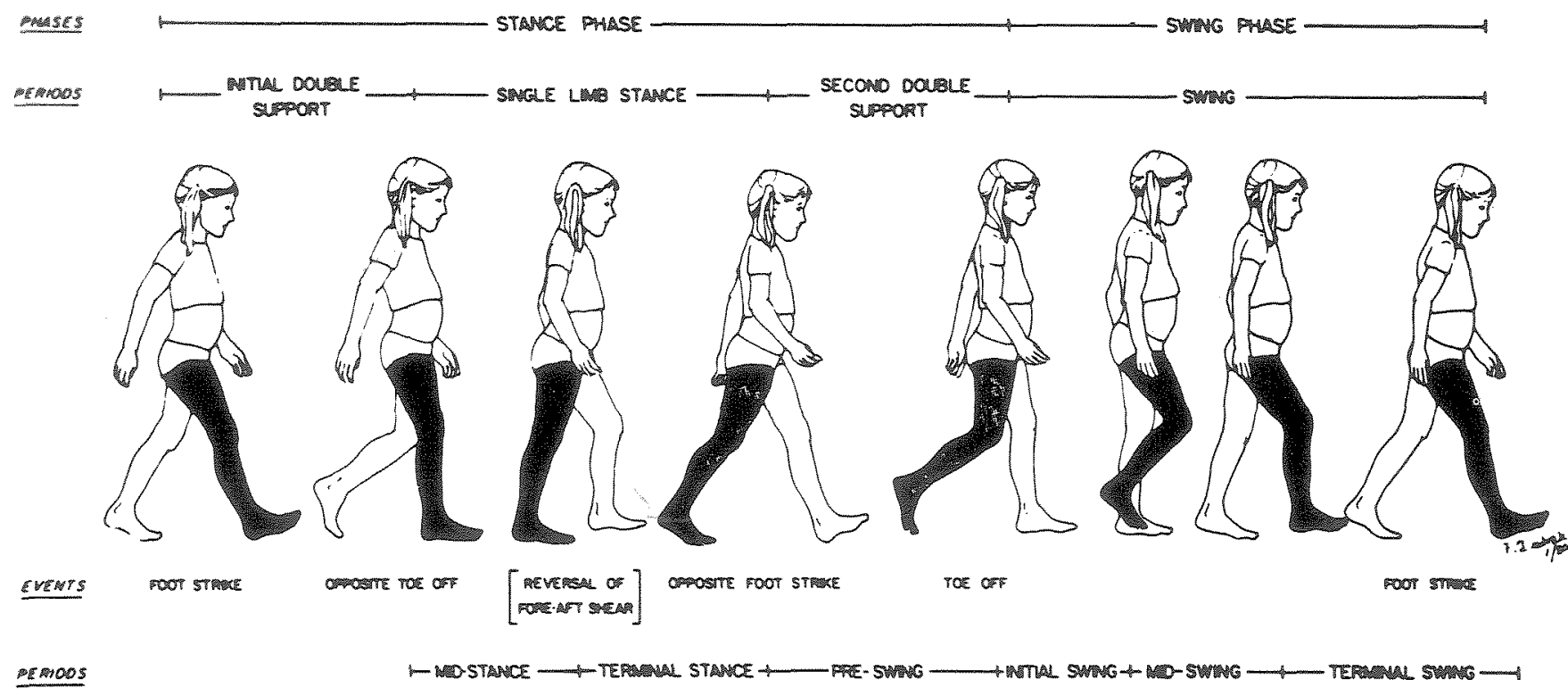

FIGURE 2

Representation of typical stride states for free speed walking. [From Sutherland (38).]

started with a presentation either of the results of a particular, usually often-quoted, investigator $o r^{\circ}$ of a consensus of consistent results. The figures in this review are primarily selected or adapted from publications that focus on many individual muscles in a particular functional group, e.g., adductors. The other investigators who have contributed to the knowledge of their function are indicated in the figure legends and references. The adapted figures are general estimates of consistent results from the referenced publications. Because of the varieties of representation mentioned previously, no attempt was made to quantitatively average any patterns. However, all of the pattern variations are discussed.

\section{Intrinsic Muscles of the Foot}

The intrinsic muscles of the foot have been studied by several investigators. Mann and Inman (18), who studied six simultaneously, have studied the most; their results are shown in Figure 3. All investigators except Sheffield et al. (29) used wire electrodes, and all of their subjects walked at free speed; the actual speeds were not whorted. The number of subjects ranged be- tween 10 and 20.

These muscles are primarily active during terminal stance and unloading periods. In persons with pronated (flat) feet, the activity of the abductor hallucis, flexor hallucis, and flexor digitorum brevis muscles began earlier in the stride, sometimes at foot-strike. The only inconsistent result is that the extensor digitorum brevis can be active throughout the stride in some individuals (29). This may be due to the surface electrode measurement or the slow speed of walking; a stride time of 2.0 seconds was used.

It is concluded that the intrinsic muscles stabilize the foot and enable it to act as a functional unit.

\section{Ankle Dorsiflexors}

Figure 4 presents a synopsis of the activity and function of the pretibial muscles, especially the tibialis anterior, which have been documented. Only Sheffield et al. (28), Rozin et al. (27), and the University of California group (39) have studied all three simultaneously. Interestingly, they used intramuscular electrodes and 10 or fewer subjects.

In general, the pretibial muscles are active 


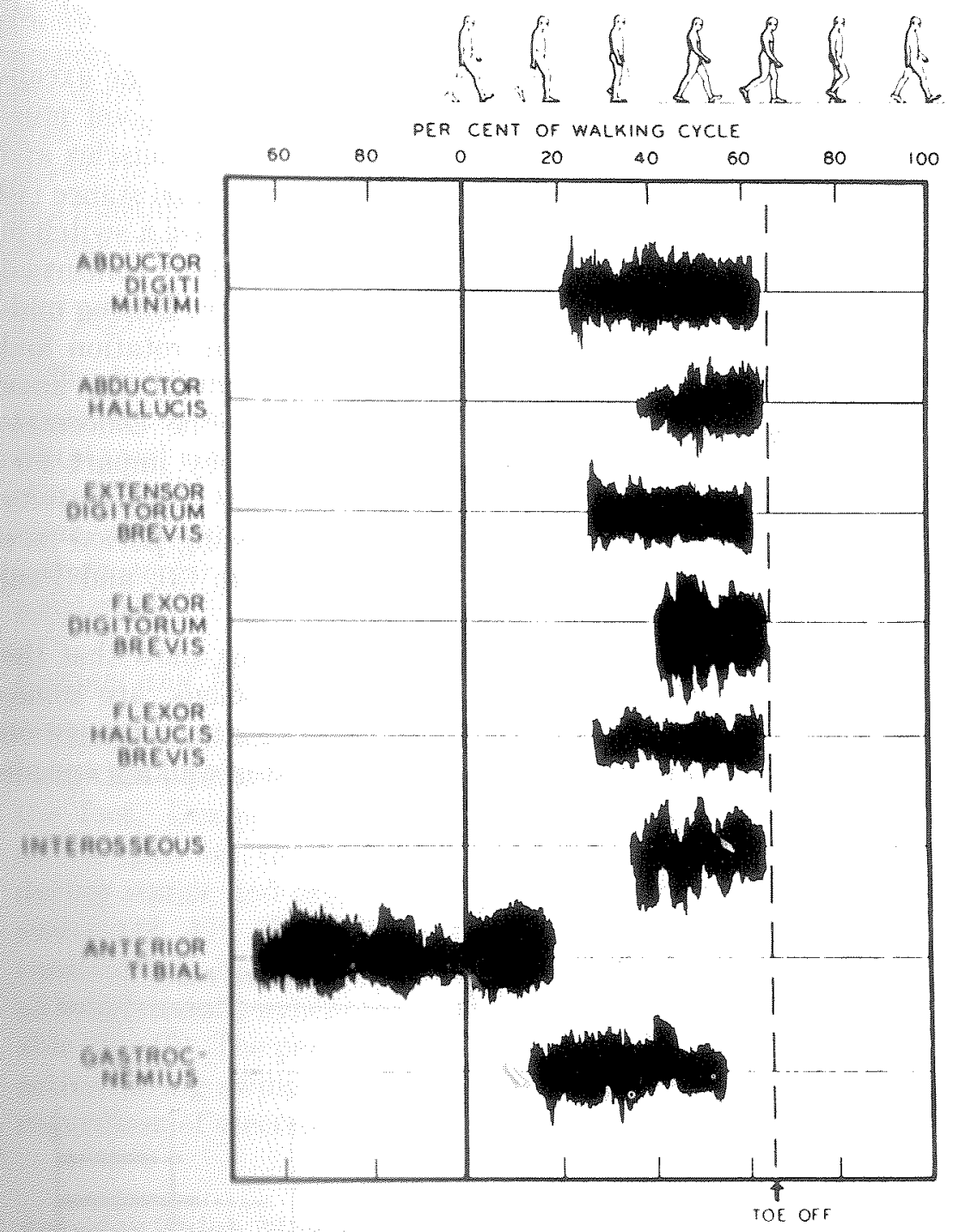

FIGURE

EMG activity of intrinsic muscles of the foot. All of the EMG is presented in measured form. Reference sources for data: abductor digiti minimi (18); abductor hallucis $(3,11,18,29)$; extensor digitorum brevis $(18,29)$; flexor digitorum brevis $(3,11,18,29)$; flexor hallucis brevis and interosseus (18). [From Mann and Inman (18).]

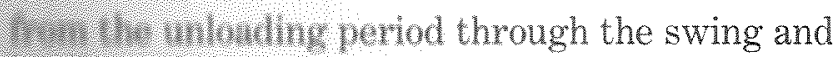
- IErols, Their activity in midswing is - pat ate und can be nonexistent in some

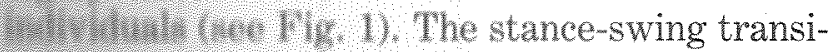
- (2) witrols hoor clearance by causing

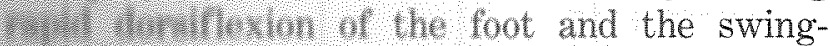

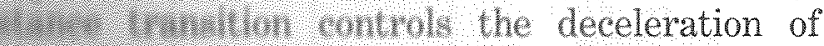
- W Int wtwe. The concentric con4. (3) Entevin, contribute to ankle - Uly Hili untorior, being also an 4te the trunder of weight to

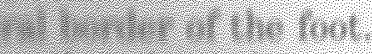

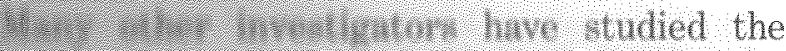
- variability during midswing and show additional variability during SLS (9, 21, 31). Basmajian (3) indicates that SLS period activity exists in those with pronated feet. It appears that the biphasic EMG pattern tends to occur more often at faster walking speeds (33).

\section{Ankle Plantarflexors}

The function of the plantarflexors has been investigated quite extensively and under almost all variations of the experimental paradigms. The triceps surae received the most attention, whereas the toe flexors received the least. Only Sutherland (36) has studied all of them simultaneously; Figure 5 presents his results and a consensus of on-off patterns for free speed walking. 
ANKLE

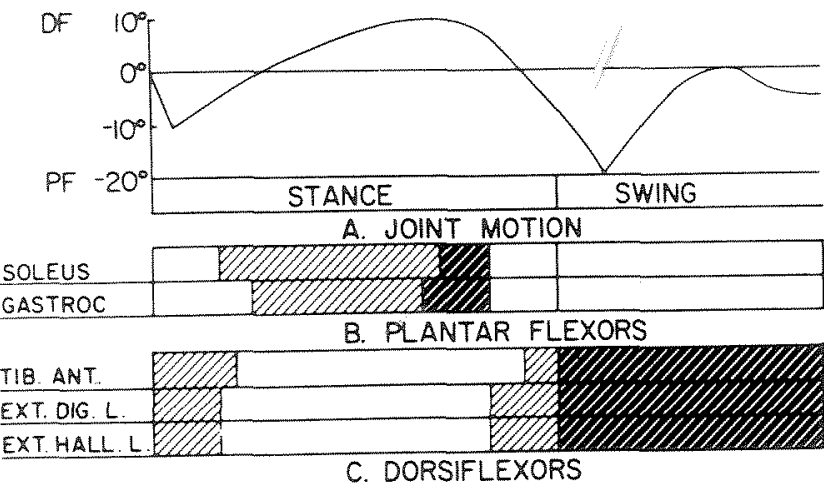

\section{FIGURE 4}

On-off patterns of EMG activity of ankle plantar flexors $(B)$ and dorsiflexors $(C)$. A normal ankle-joint angle trajectory depicting dorsiflexion (DF) and plantarflexion (PF) is also shown $(A)$. Reference sources for data on dorsiflexors: tibialis anterior $(4,9,11,19,27,29,31,39)$; extensor digitorum longus $(29,39)$; extensor hallucis longus $(27,29,39)$; group (10). [From Perry (23).]

\section{Triceps Surae}

All the plantarflexor muscles, with the exception of the gastrocnemius, are single-joint muscles. They are mainly stance-phase muscles and all contract eccentrically until terminal stance ( 40 percent of gait cycle), when they have a switch in function. The triceps surae are mainly concerned with control of forward momentum. During midstance they decelerate tibial-talar rotation and allow forward momentum to extend the knee joint. At the end of midstance the concentric contraction phase begins and they impart forward momentum (i.e., push-off) (36). Activity usually ceases during the unloading period [see Eberhardt et al. (10); Basmajian (3); and Paul (21)].

The more recent investigations show that the triceps surae, more often the soleus, are sometimes active during the swing-stance transition period $(9,27)$ especially at faster walking speeds

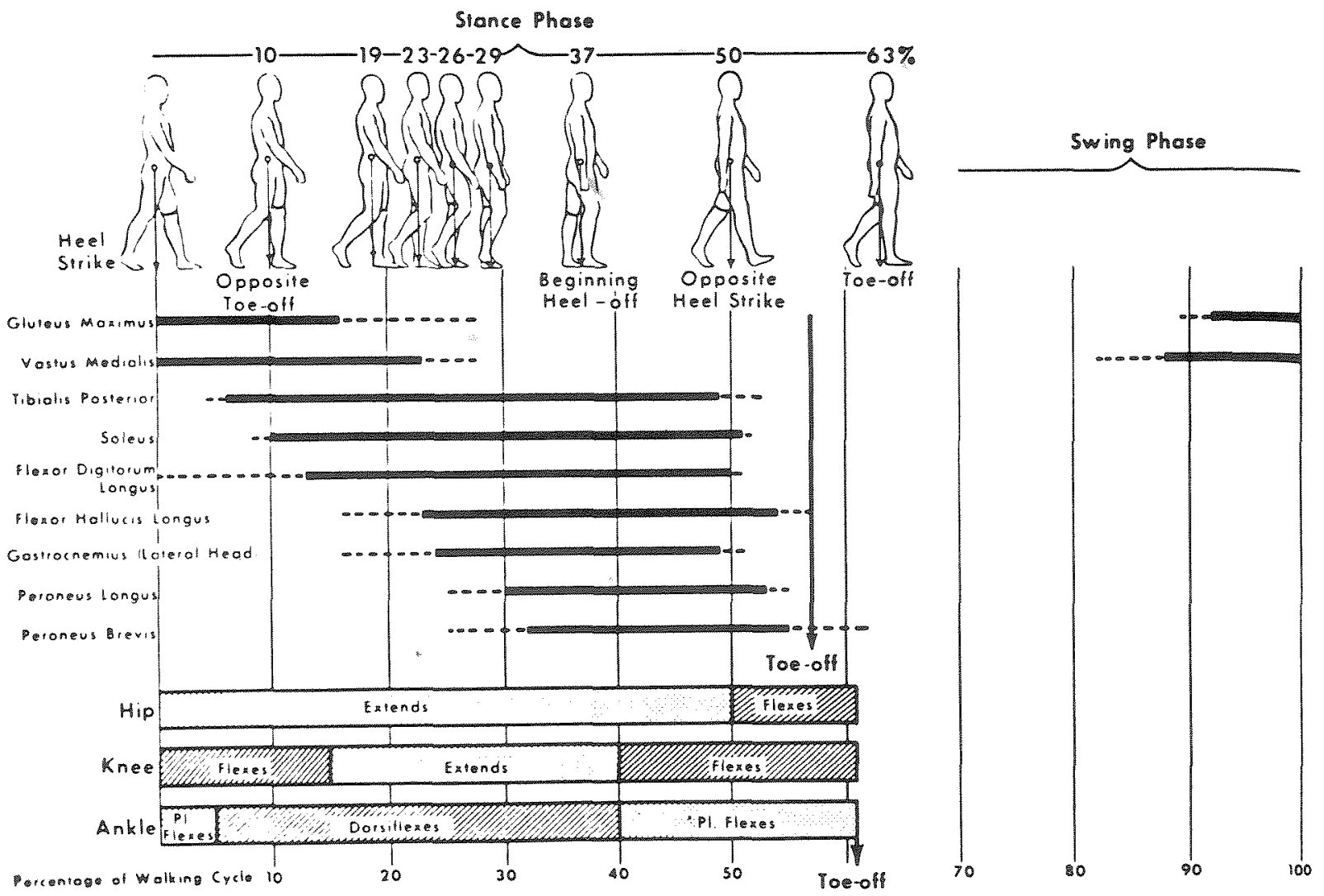

\section{FUCU日e 5}

Onwof patterns of EMG activity of ankle plantarflexion. Generd motions of leg joints are also indicated. Reference

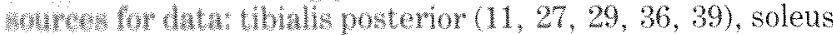

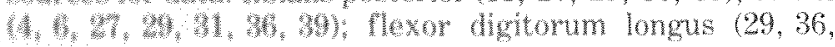

39); flexor hallucis longus $(11,29,36,39)$; gastrocnemius $(6,9,19,27,29,31,36,39)$; peroneus longus $(11,27,29$, $31,36,39,40)$; peroneus brevis $(36,39,40)$; group (10). [From Sutherland (36).] 
$(6,19,34)$. The activity is still monophasic. The eccentric contraction of the gastrocnemius during the deceleration stage at faster speeds contributes to the deceleration of the tibia. Figure 6 shows the change in timing of the gastrocnemius as a function of walking speed; soleus changes are similar (34).

\section{Toe Flexors}

The function of the long toe flexors seems to be primarily to stabilize the toes during the time of metatarsal contact, since their size would imply that there is little contribution to plantarflexor moment. Their timing has been investigated with intramuscular electrodes only at free speed and is consistent.

\section{Peronei and Tibialis Posterior}

The peronei and tibialis posterior are antagonists that primarily control inversion-eversion of the foot. During walking they control weight distribution on the lateral border of the foot during stance and orientation of the foot during swing $(12,40)$. The tibialis posterior is fairly consistent in its activity; whereas, the peronei, although usually synchronous, are definitely variable $(29,40)$. The peronei in many individuals are active during swing-phase and transition periods regardless of electrode type and walking speed.

\section{Anterior Thigh Musculature}

Figure 7 shows a typical estimate of the patterns of activity for free speed of the muscles located in the anterior thigh (26). The quadriceps, except for the vastus intermedius, have undergone intense investigation. The others have not, probably because of either their relative unimportance for essential function or difficulty of measurement. There is much variation both intra- and inter-individually.

\section{Quadriceps}

The quadriceps are consistently active during the swing-stance transition period. There is much variability during the remainder of the stride, and it does not matter which type of electrodes are used. The activity of the vastus intermedius is synchronized with the other vasti (1). The predominant variation is the activity during the stance-swing transition period as depicted in Figure 1. The collation of the results of many investigations show the following general trends: 1) at free speed, approximately 67 percent of the rectus femoris and 45 percent of the vasti patterns show activity during the stance-swing transition period; 2) the frequency of occurrence of the latter activity correlates with walking speed; 3 ) the major phase of activity of the quadriceps tends to elongate into midstance as walking speed increases in approximately 40 percent of the patterns. Interestingly, Brandell's (6) results are the only ones showing the vastus medialis having predominantly midstance and terminal stance activity; his subjects walked on a treadmill.

The first phase provides knee stability for loadbearing and ironically ceases activity when knee extension commences. The second phase of actu.

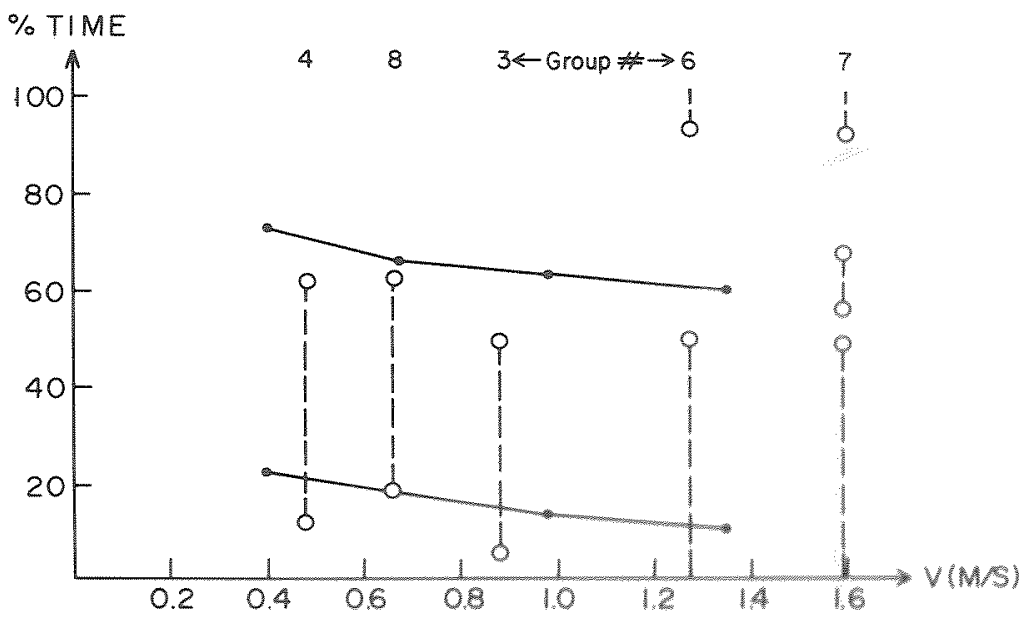

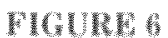

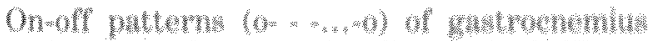

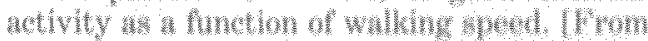

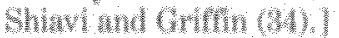




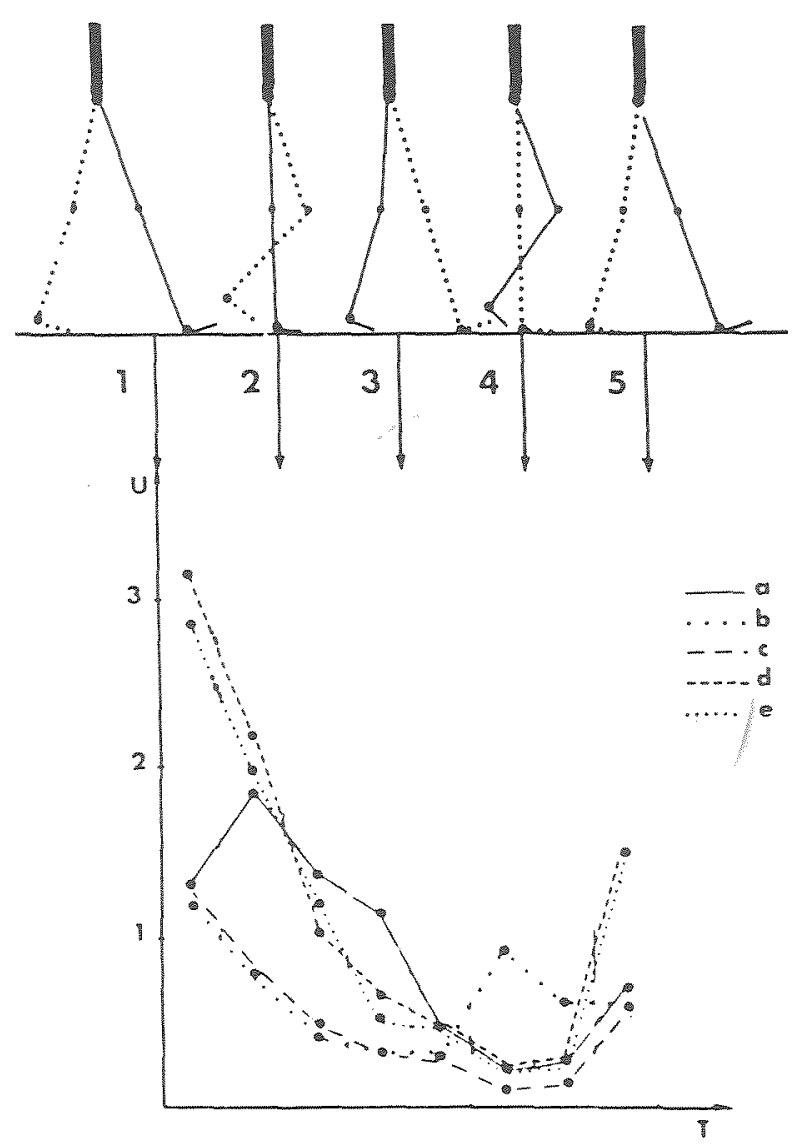

FIGURE 7

Patterns of activity of anterior knee musculature. Reference sources of data for $a$ : tensor fasciae latae and $b$ : sartorius $(3,26,39)$; $c$ : rectus femoris $(21,26,31,39)$; $d$ : vastus medialis $(1,6,26,27,39)$; $e$ : vastus lateralis $(1,9,19,26,27$, $31,39)$; vastus intermedius $(1,39)$; group $(4,10)$. [From Rideau and Duvall (26).]

FIGURE 8

On-off patterns of consistent EMG activity of posterior knee musculature. Reference sources for data: biceps femoris brevis $(3,39)$; biceps femorus longus $(3,17,39)$, semimembranosis $(3,9,17,19,21,31,39)$; semitendenosis $(3,9,19$, $21,31,39)$; group $(4,10)$.

\section{FIGURE 9}

On-off patterns of consistent EMG activity of single-joint hip extensor/flexors. Reference sources for data: gluteus maximus $(3,4,10,17,39)$; iliopsoas $(3,4,21,39)$. ity occurs primarily during the loading stage of the opposite extremity. It may help stabilize the knee and is associated with acceleration of the leg at the onset of swing.

\section{Sartorius}

The sartorius can be biphasically active during loading and initial swing (26). Only Basmajian (3) does not report a loading period action. The initial swing activity elongates into the unloading period with faster walking speed (39). This latter activity contributes to hip flexion.

\section{Posterior Thigh Musculature}

The hamstrings comprise this group and have been studied under a variety of conditions: separately, as medial and lateral groups, and with surface or wire electrodes, etc. Figure 8 is an adaptation from the indicated investigators and shows the most consistent activities.

All heads of the hamstrings are active within the swing-stance transition period. However, the duration of the swing-phase portion at slower speeds of walking changes with walking speed. As speed increases from slow to fast, the swingphase activity elongates from terminal swing period into the initial swing period $(17,31)$. An inconsistent second phase during the stanceswing transition period. (see Fig. 1) was found by the majority of investigators in approximately 40

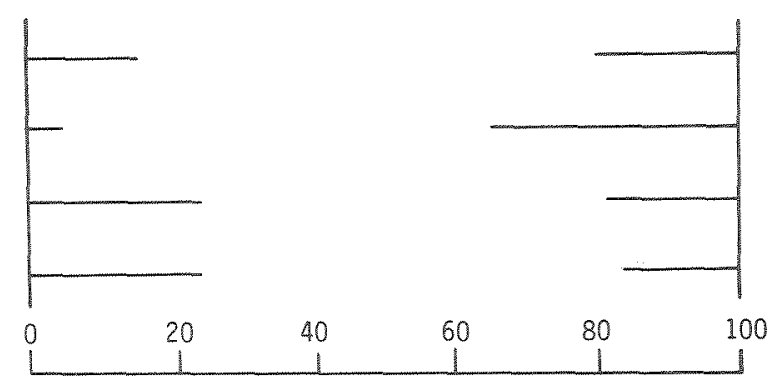

Gluteus Maximus

11 iopsoas

$\%$ Stride
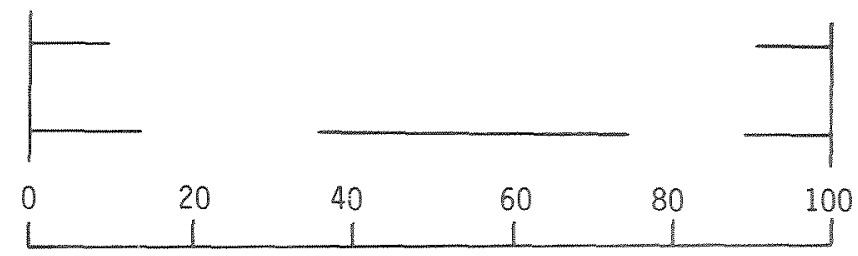
percent of the subjects. A level of activity of the medial hamstrings during most of midstance has also been reported by Paul (21), whose subjects walked only in the fast speed range: 1.4 to 2.1 meters per second. However, Milner et al. (19) did not find this activity at speeds of 2.28 meters per second.

In the consistent phase these muscles undergo a switch in function. The well-known deceleration of the lower extremity is accomplished in late swing with eccentric contraction. At heel-strike the muscles contract concentrically and hence cause hip extension during the loading and midstance periods. The inconsistent second phase is also an eccentric contraction, occurring while the hip joint is flexing. This action serves to assist knee flexion and leg clearance.

\section{Popliteus}

The activity of the popliteus has been reported by Perry (23). It is active during midstance and seems to contribute to deceleration of knee-joint extension.

\section{Hip Joint Musculature}

\section{Hip Extensor}

The gluteus maximus is the primary singlejoint hip extensor. It is consistently active during the swing-stance transition period and contributes initially to hip-joint stabilization and then to hip extension (Fig. 9). An inconsistent second phase of smaller magnitude during the stanceswing transition period also exists $[(3,4,10)$; see Fig. 1]. Given that hip flexion is occurring, this second phase would contribute to pelvic stabilization.

\section{Hip Flexor}

The iliopsoas is the primary single-joint hipflexor muscle. Its activity from terminal stance through midswing is a consistent finding. From a small study it seems that the duration is shorter in women (4). However, several investigators have claimed that another phase of activity exists during the swing-stance transition period. Since these latter investigators used surface electrodes in the femoral triangle, this second phase could be the result of crosstalk $(4,21)$. Figure 9 shows this summary.

The iliopsoas actually shows a switch in function during its acknowledged range of activity. During stance it is lengthening and must be assisting hip stabilization. During swing it is flexing the hip and becomes quiescent when the hip-joint angle becomes constant. If the questionable phase of activity is accurate, the exact function is difficult to discern because of the multitude of hip muscles that are active during that time.

\section{Abductor Group}

Figure 10 and Figure 1 show a consensus of

Tensor Fasciae Latae
Gluteus Medius
Gluteus Minimus
$\%$ Stride
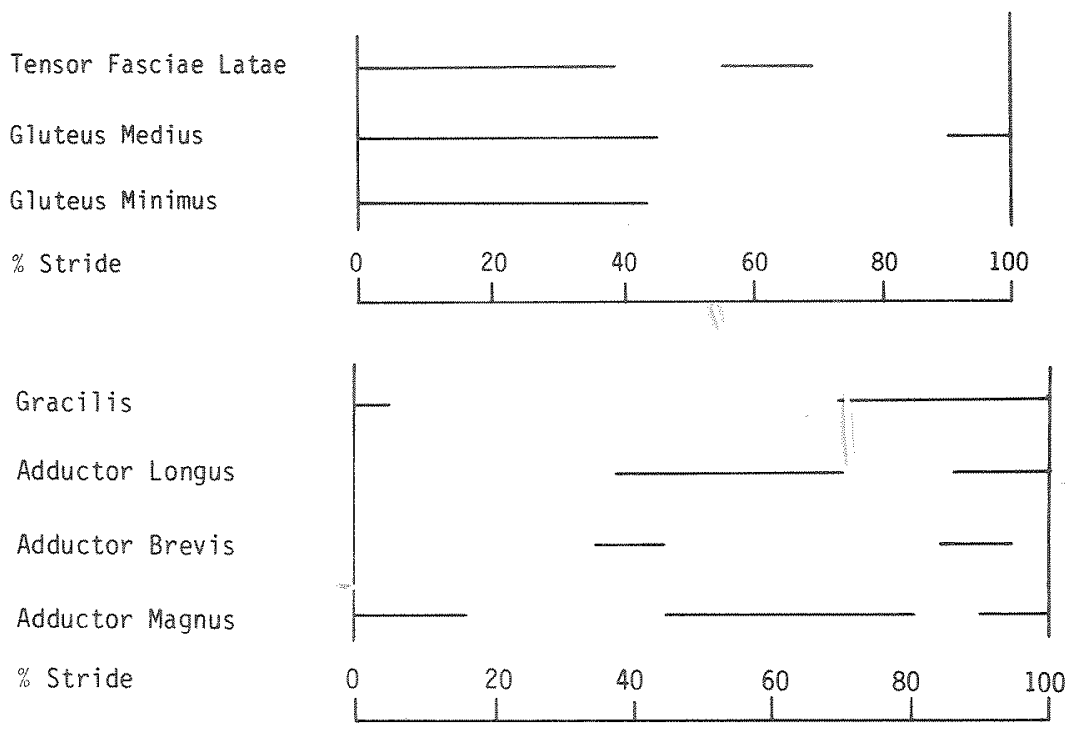

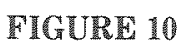

On-off patterns of consistent EMG activity of abductors. Reference sources for data: tensor fasciae latae $(3,17,26,39)$; gluteus medius $(3,17,39)$; gluteus minimus $(3,39)$; group $(4,10,21,31)$.

\section{FIGURE 11}

On-of patterns of consistent EMG activity of adductors. Reference sources for data: gracilis and adductor longus $(3,39)$; adductor brevis (3); adductor magnus $(3,12,21,39)$; group $(4,10)$. 
patterns for the abductor muscles. The results of those who studied the gluteus medius using surface electrodes are merged into the group pattern.

The gluteus medius and minimus are active in everyone from loading through midstance and cease activity prior to loading of the opposite extremity. As walking speed increases, this phase for the gluteus medius extends into terminal swing in a greater percentage of people (17, 31). This activity stabilizes the pelvis in the frontal plane during stance. A second phase exists in approximately 40 percent of people during the stance-swing transition period $(3,31)$. This causes a slight abduction to promote leg clearance. There have been few observations of isolated gluteus medius activity during midswing $(4,17)$. The gluteus minimus also has a second phase of activity, but it occurs during midswing (3).

The tensor fasciae latae has been reported to show two distinct patterns of activity. One group of investigators report a biphasic pattern as shown in Figure 10. The other group reports a monophasic pattern in midstance. Both pattern types were recorded with wire electrodes. Functionally, the tensor fasciae latae is generally an abductor and additionally may control the rotation of the femur (3).

\section{Adductor Group}

A few of the older articles report the activity of the adductor group, i.e., adductors and gracilis. A synopsis is presented in Figure 11.

The three reports on the gracilis are perplexing although all used wire electrodes. Paul (21) shows it monophasically active through most of swing phase and loading priod, Basmajian (3) reports it more like the adductor longus pattern in the figure, and the UC group (39) reported both types of activity. Presently there is no explanation for this duality.

The adductors can be biphasically active near

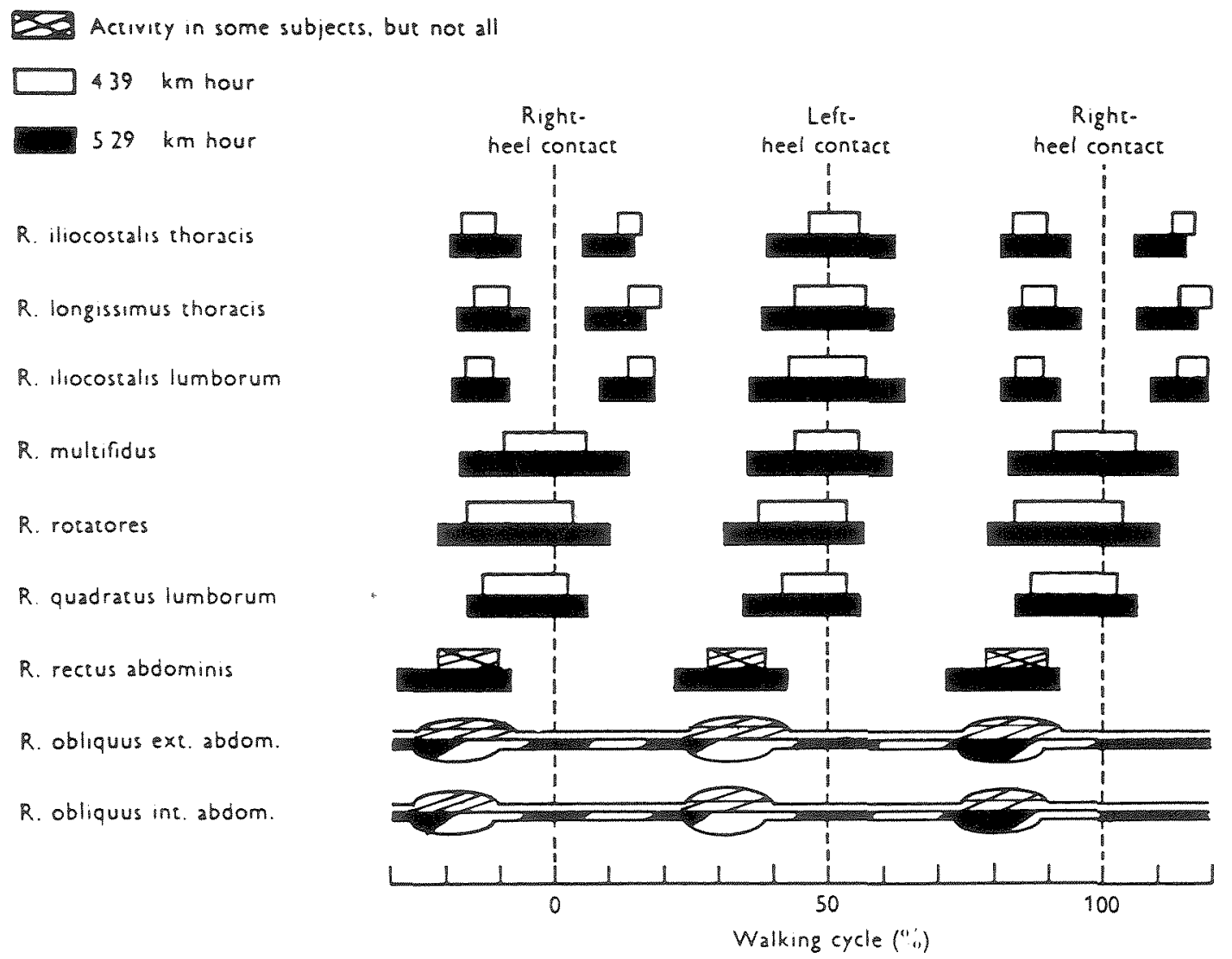

FIGURE 12

EMG activity of trunk muscles. [From Waters and Morris (41).] 


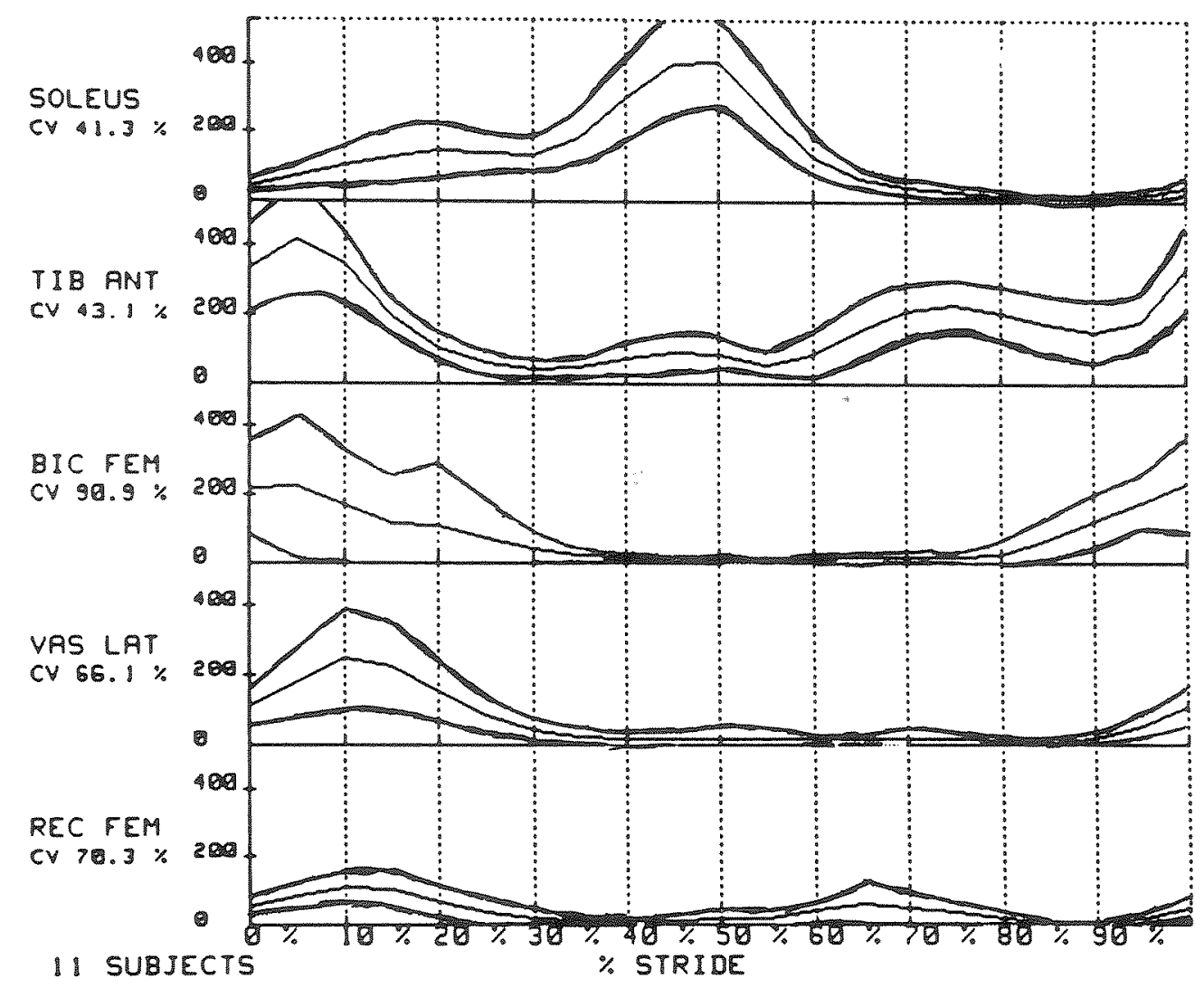

FIGURE 13

Ensemble average (middle curves) and one standard deviation band (outer curves) of EMG envelopes from 11 normal subjects walking at free speed. [From Winter (43).]

to or during the transition periods. Both of these phases correspond to the times when the direction of transverse rotation of the pelvis is changing (10). The stance-swing transition phase is also thought to contribute to lateral rotation of the femur. The adductor magnus is functionally two muscles. The upper portion is a true adductor, whereas the lower portion functions as a hamstring muscle (3). It essentially has the biphasic activity shown and reflects the group activity. The exception is the treadmill study by Green and Morris (12), whose results do not show any stance-swing transition activity. Only Basmajian (3) reports on the adductor brevis. Its pattern varies with the speed of walking, and at free speed it has a biphasic pattern. The variations are not documented.

\section{Trunk Musculature}

There has been very little investigation into the activity of the trunk musculature during level walking. Figure 12, from Waters and Morris (41), presents a summary of currently known EMG patterns.

\section{Abdominal}

The abdominal muscles (rectus abdominus, external oblique, internal oblique) are very variable in their activity $(28,41)$. The latter two are continuously active at free speed and have superimposed phases at faster speed as shown: The rectus abdominus is active in only 50 percent of subjects at free speed $(\mathrm{ST}=1.2 \mathrm{~s})$ and always phasically active at faster speeds $(\mathrm{ST}=1.0 \mathrm{~s})$. This seems to be consistent with Sheffield (28), who reports no activity; however, his subjects were walking slowly with a stance time equaling 2 seconds.

\section{Paraspinal}

Even though the paradigms were diverse (floor and treadmill, wire and surface electrodes, ete.) 
Journal of Rehabilitation Research and Developm nol. 22 No. 3 July 1985

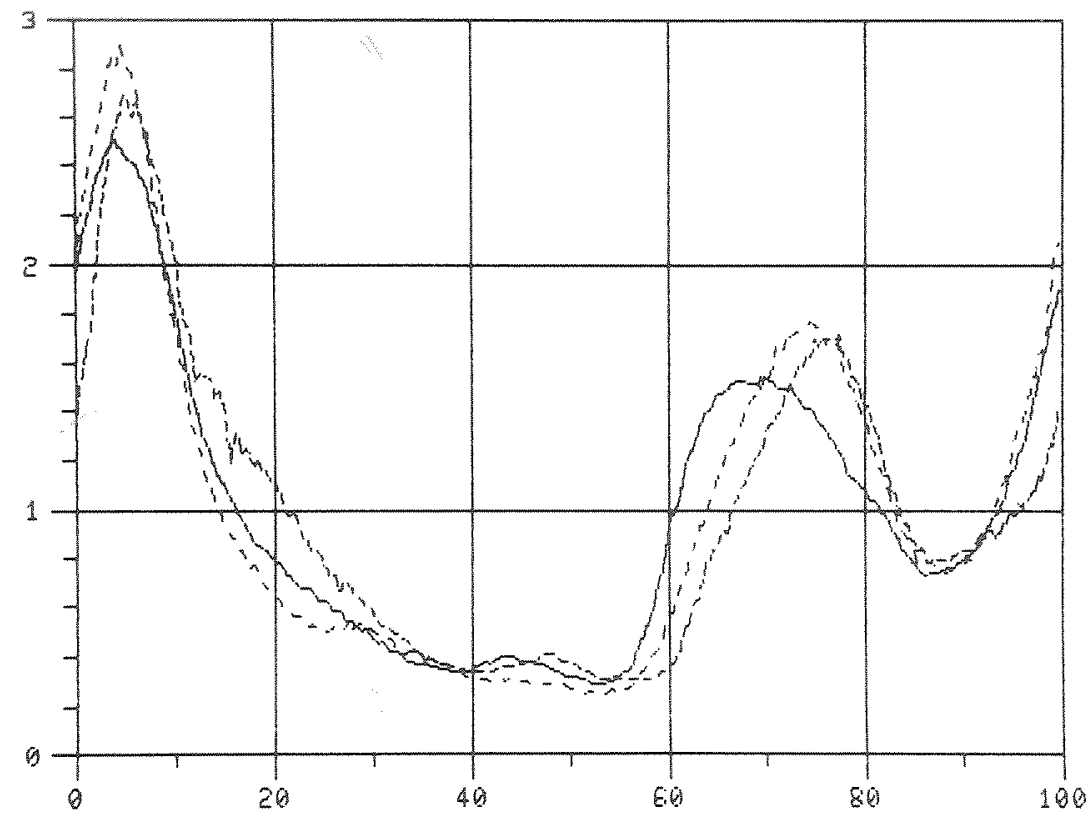

PIGURE 14

Ensemble averages of EMG envelopes from tibialis anterior muscle for three speed ranges: slow (- ), free (- -), fast (-).

the results are similar $(4,10,39,41)$. The paraspinal muscles are active during the transition periods at free and fast speeds. The only inconsistency is that the erector spinae is not always active during the swing-stance transition at free speed (41). A major difference is that the wire electrode recordings showed two bursts of EMG during the stance-swing transition, whereas the surface recordings showed continuous activity. The stance times were equal for free speed.

The transitional period activities coincide with the time when the pelvic and thoracic rotations are changing direction and the weight support is being shifted. Note that the phases differ in timing by 50 percent; that is, the first phase of the ipsilateral extremity has the same timing as the second phase of the contralateral extremity. Since all of these muscles exist bilaterally, these muscles are contracting bilaterally, one concentrically and the other eccentrically, performing a stable balancing of the torso and pelvis and elevating the pelvis in preparation for swing phase.

\section{CONCLUSION}

All of this information needs to be effectively compiled into a useful data base for comprehensively understanding the variations in EMG gait patterns and for providing a normative data base to evaluate locomotor deficits $(15,37)$. The quantitative form of individual patterns in the data base must be the average envelope from several strides. Although there is a high intra-subject repeatability of patterns, some stride-to-stride variation exists (2). Recently, Winter (43) has shown the validity of using EMG profiles for normal versus pathologic comparisons. The profiles are formed with the ensemble average and variation curves of the linear envelopes of a sample from the normal population. Figure 13 shows such profiles. Shiavi et al. (35) have advocated such an approach and have used it to evaluate pattern variations in childhood gait.

Normalization, which is a general problem, still needs to be resolved before this concept can be effectively implemented. As mentioned previously, amplitude normalization with the pattern average reduces the variation more than other amplitude normalization parameters. However, further time-base normalization is required. Figure 14 shows the ensemble averages of the tibialis anterior muscle's EMG pattern for 25 normal adults sorted by walking speed, which means 
stride times are also different. If the stride is segmented such that the stance and swing phases have equal normalized durations, the pattern alignment would be better and hence the variation would be reduced. This is a rational procedure since functional regions of gait become

\section{REFERENCES}

1. ADLER N, KENT B: Electromyography of the vastus medialis in normal subjects during gait. 4th Congress of International Society of Electrophysiological Kinesiology, Boston, 1979 .

2. Arsenault B, Winter D, Wells R: Repeatability of electromyographic activity during gait. Human Locomotion-1-Canadian Society for Biomechanies Conference, London, Ontario, September 1980.

3. Basmajian JV: Muscles Alive. Baltimore, MD: Williams \& Wilkins, 1978.

4. BATTYE C, JosepH J: An investigation by telemetering of the activity of some muscles in walking. Med Biol Eng 4: 125-135, 1966.

5. Bodem F, Brussatis F, Wunderlich T, Mertin B: A kinesiologic electromyography system for the computer-controlled analog and digital recording and processing of muscle action potentials of walking subjects. Med Prog Tech 8: 129-139, 1981.

6. BRANDELL B: Functional roles of the calf and vastus muscles in locomotion. Am J Phys Med 56:59-74, 1977.

7. DEAN G: An analysis of the energy expenditure in level and grade walking. Eroonomics 8: 31-48, 1965.

8. DRILLIS R: The influence of aging on the kinematics of gait. In: The Geriatric Amputee. Washington, DC: National Academy of Sciences-National Research Council, 1961.

9. Dubo H, Peat M, Winter D, Quanbury A, Hobson D, STEINKE T, REIMER G: Electromyographic temporal analysis of gait: normal human locomotion. Arch Phys Med Rehabil 57: 415-420, 1976.

10. EBERHART H, INMAN V, BRESLER B: The principal elements in human locomotion. In: Human Limbs and Their Substitutes. P. Klopsteg and P. Wilson (eds.). New York: Hafner, 1954.

11. GRAY E, BASMAJIAN J. Electromyography and cinematography of leg and foot ("nomal" and "flat") during walking. Anat Rec 161: 1-15, 1968.

12. GREEN D, MorRIS J: Role of adductor longus and adductor magnus in postural movements and in ambulation. Am I Phys Med 49: 223-240, 1970.

13 GrIEvE D: Gait patterns and the speed of walking. Biomed Eng 3: 119-122, 1968.

14. GRIEVE DW, CAVANAGH PR: The validity of quantitative statements about surface, electromyograms recorded during locomotion. Scand J Rehabil Med 3. 19 25,1974 .

15. GUTH V, ABINK F, THEYSOHN H: Electromyographic investigations on gait. Electromyograph Clin Neurohysiol 19: 305-323, 1979.

16. INMAN V, RALSTON H, TODD F: Human Walking. Baltimore, MD: William \& Wilkins, 1981.

17. LYONS K, PERRYJ, GRONLEYJ, BARNES L, ANTONEL- aligned. Perry et al. (25) suggested and used this when studying on-of patterns in hemiplegic gait. The UC group (39) also presented their data in this manner. Definitive analyses need to be undertaken to find the optimal normalization procedures.

LI D: Timing and relative intensity of hip extensor and abductor muscle action during level and stair ambulation. Phys Ther 63: 1597-1605, 1983.

18. MANN R, INMAN V: Phasic activity of intrinsic muscles of the loot: I Bone Joint Surg 406A: 469 481, 1964.

19. Milner M, BAsmajtan J, Quanbury A: Multifactorial analysis of walking by electromyography and computex. Am J Phys Med 50: 235-258, 1971.

20. Milner M, Quanbury A: Facets of control in human walking. Nature London 227:734-735, 1970.

21. PAUL J: Comparison of EMG signals from leg muscles with the corresponding force actions calculated from walkpath measurements. In: Human Locomotor Engineering. London: Inst Mech Eng, 1974.

22. PEDorT A: A study of motor coordination and neurom muscular activities in human locomotion. Biol Cyber 26:53-62, 1977

23. PERRY J: Kinesiology of lower extremity bracing. Clin Orthot Rel Res 102: 18-31, 1974.

24. PERRY J: Generic terminology for the phases of gait. Bull Pros Res 18: 279-281, 1981. (Gait Analysis Workshop, Rehabilitative Engineering Research and Development Service, Veterans Administration, Long Beach, CA, September 1979.)

25. PERRY J, WATERS R, PERRIN T: Electromyographic analysis of equinovarus following stroke. Clin orthot Rel Res 131: $47-53,1978$.

26. RIDEAU Y, DUVAL A: Function of the anterior thigh muscles. Anat Clin 1: 29-42, 1978.

27. RozIN R, ROBIN G, MAGORA A, SIMKIN A, GONEN B: Investigation of gait-2. Gait analysis of normal individuals. Electromyography 12: 183-190, 1971.

28. SHEFFIELD F: Electromyographic study of the abdominal muscles in walking and other movements. Am. Phys Med 41: 142-147, 1962.

29. ShefField F, Gersten J, MAstellone A: Electromyographic study of the muscles of the foot in normal walking. Am J Phys Med 35: 223-236, 1956.

30. SHIAVI R, BOURNE J, HOLLAND A: Automated extraction of activity features in linear envelopes of locomotor electromyographic pattems. In press.

31. SHIAVI R, CHAMPION S. FREEMON F, GriffiN P: Variability of electromyogaphic patterns for level surface walking through a range of self-selected speeds. Bull Prosthet Res 18: 5-14, 1981.

32. SHIAVI R, GREEN N: Ensemble averaging of locomotor electromyogaphic patterns using interpolation. Med Biol Eng Comput 21: 573-578, 1983.

33. SHIAVI R, GRIFFIN P: Representing and clustering electromyographic gait patterns with multivariate techniques. Med Biol Eng Comput 19: 605-611, 1981.

34. SHIAVI R, GRIFHIN P: Changes in electromyographic gait patterns of calf muscles with walking speed. $I E E E$ Trans Biomed Eng 30: 73-76, 1983 . 
35. ShIAVI R, MCFADYEN B, GREEN N: Electromyographic patterns in normal pediatric gait. IX International Congress of Biomechanics, August 1983, Waterloo, Ontario.

36. SUTHERLAND D: An electromyographic study of the plantar flexors of the ankle in normal walking on the level. I Bone Joint Surg 48A: 66-71, 1966.

37. SUTHERLAND D: The value of normative data in gait analysis. Gait Research Workshop. U.S. Department of Health, Education, and Welfare, 1978, pp. 89-128. (NIH 78-119.)

38. SUTHERLAND D: Events measurements in normal and pathological gait. Bull Prosthet Res 18: 281-282, 1981. (Gait Analysis Workshop, Rehabilitative Engineering Research and Development Service, Veterans Administration, Long Beach, CA, September 1979.)

39. UNIVERSITY OF CALIFORNIA, BERKELEY: The pattern of muscular activity in the lower extremity during walking. Advisory Committee on Artificial LimbsNational Research Council; Prosthetics Devices Re- search Project Inst Engr Res Report Series II Issue 25, September, 1953.

40. WALMSLEY R: Electromyographic study of the phasic activity of peroneus tongus and brevis. Arch Phys Med 58: 65-69, 1977

41. WATERS R, MORRIS J: Electrical activity of muscles of the trunk during walking. $J$ Anat 111: 191-199, 1972.

42. Winter D, RAU G, Kadefors R, Broman H, DeLUCA C: Units, terms, and standards in the reporting of EMG research. International Society of Electrophysiological Kineosiology, August 1980. (Report of Ad Hoc Committee.)

43. WINTER D: Pathologic gait diagnosis with computeraveraged electromyographic profiles. Arch Phys Med Rehabil 65: 393-398, 1984.

44. YANG J, WINTER D: The effect of EMG normalization methods on intersubject variability. Canadian Society for Biomechanics Conference, September 1982, Waterloo, Canada. 